Z. klin. Chem. u. klin. Biochem.

9. Jg., S. 257-265, Mai 1971

\title{
Zur Methodik der radioimmunchemischen Bestimmung von menschlichem thyreotropen Hormon (H-TSH) ${ }^{1}$ )
}

\author{
Von A. v. zUR MÜHLEN und D. EMrICH
}

\begin{abstract}
Medizinische Universitätsklinik (Arbeitsgruppe Endokrinologie) und Nuklearmediziniscbe Abteilung der Medizinischen und Radiologischen Universitätsklinik Göttingen
\end{abstract}

(Eingegangen am 21. Dezember 1970)

\begin{abstract}
Es wird über methodische Untersuchungen zur radioimmunchemischen TSH-Bestimmung in verschiedenen Systemen und mit mehreren Hormon- und Antikörperpräparationen berichtet. Es gelang nicht, mit Hilfe eines heterologen B-TSH-Systems (hochgereinigtes B-TSH und B-TSH-Antiseren eigener Herstellung) thyreotropes Hormon (Plasma; Human Thyrotropin Research Standard A) nachzuweisen. Dagegen konnte H-TSH (nicht jedoch B-TSH) mit homologen Systemen (NIH; Firma Calbiochem) unter Verwendung verschiedener H-TSH-Präparationen (Hypothyreoten-Plasma; TSH-Präparationen: NIH, Firma Calbiochem; Human Thyrotropin Research Standard A) dosisabhängig bestimmt werden: Empfindlichkeit 1,5-2 $\mu \mathrm{U} / \mathrm{m} l$ Plasma; Genauigkeit $\pm 5 \%$; Reproduzierbarkeit $\pm 10 \%$ im höheren und $\pm 20 \%$ im niederen Konzentrationsbereich. Die Verwendung von vorzugsweise mit ${ }^{131} \mathrm{~J}$-markiertem Hormon gleichbleibend hoher spezifischer Aktivität ist für die Reproduzierbarkeit der Bestimmungsmethode von großer Bedeutung.

Bei $60 \%$ eines Normalkollektivs von 30 Personen und den meisten der 10 hyperthyreoten Patienten lagen die TSH-Spiegel im Plasma nach Abzug des „virtuellen“ Wertes eines Hypophysektomierten-Plasmas unter der methodischen Erfaßbarkeit. Bei 25 primär hypothyreoten Patienten wurden TSH-Spiegel von 12-600 $\mu \mathrm{U} / \mathrm{m} l$ gemessen.

Zu einer Mitreaktion von zwei LH-Präparationen (NIH; Firma Calbiochem), HCG und 2-IRP-HMG im H-TSH-System (NIH) kam es im wesentlichen nur nach dem Grad ihrer Verunreinigung durch H-TSH. Dagegen bestand eine ausgeprägte Kreuzimmunität zwischen den geprüften TSH-Präparationen und LH im radioimmunologischen LH-System (H-LH: NIH) unter Verwendung zweier verschiedener Antiseren (NIH; Firma Calbiochem).
\end{abstract}

\section{Studies on the radioimmunochemical determination of buman tbyroid stimulating bormone (H-TSH)}

The radioimmunochemical determination of TSH was studied.in different systems and with several preparations of hormone and antibody. Thyroid stimulating hormone (plasma; human thyrotropin Research Standard A) could not be detected with the aid of an heterologous bovine-TSH system (high purity B-TSH and B-TSH antisera prepared in the laboratory). On the other hand, using different H-TSH preparations (hypothyreotic plasma; TSH preparations from NIH and from Calbiochem; human thyrotropin Research Standard A), H-TSH (but not B-TSH) could be determined with homologous systems (NIH; Calbiochem): Sensitivity $1.5-2 \mu \mathrm{U} / \mathrm{ml}$ plasma; accuracy $\pm 5 \%$; reproducibility $\pm 10 \%$ in the upper concentration range and $\pm 20 \%$ in the lower concentration range. The preferential use of constant high specific activity hormone labelled with ${ }^{151} \mathrm{I}$ is very important for the reproducibility of the determination method.

In $60 \%$ of a normal collective of 30 persons and in the majority of 10 hyperthyreotic patients, the TSH level in the plasma, after subtraction of the "virtual" value of an hypophysectomised plasma, was below the limits of detection by the method. In 25 primary hypothyroid patients, a TSH level of $12-600 \mu \mathrm{U} / \mathrm{m} l$ was found.

The reaction of two LH-preparations (NIH; Calbiochem), HCG and 2-IRP-HMG in the H-TSH system (NIH) depended essentially on the degree of their contamination with H-TSH. On the other hand, there was a marked cross reaction between the tested TSH preparations and $\mathrm{LH}$ in the radioimmunological LH-system (H-LH from NIH), using two different antisera (NIH; Calbiochem).

Die bisher angegebenen biologischen Methoden zur Bestimmung von $\mathrm{H}-\mathrm{TS} \mathrm{H}^{2}$ ) im Plasma (Übersichten 1, 2) sind für den Nachweis normaler oder erniedrigter Hormonspiegel, bis auf die in vitro-Methode von KIRKHAM (3), zu unempfindlich. Ihre Einsatzfähigkeit wird darüber hinaus durch den großen methodischen Aufwand, die erhebliche Streubreite und die Beeinflussung durch unspezifische Faktoren weiter eingeschränkt. UTIGER (4) und ODELL (5) beschrieben erstmals eine radioimmunchemische Nachweismethode für H-TSH, wobei eine hochgereinigte H-TSH-Präparation sowohl für die Radiojodmarkierung als auch zur Erzeugung von Anti-

1) Mit Unterstützung der Deutschen Forschungsgemeinschaft, Bad Godesberg.

2) Abkirzungen: H-TSH $=$ Menschliches, B-TSH $=$ Rinder-, P-TSH = Schweine-Thyreotropes-Hormon; H-LH = Menschliches luteinisierendes Hormon; HCG = Menschliches Choriongonadotropin. körpern Verwendung fand. Zur gleichen Zeit publizierten Lemarchand-Beraud und Vanotti (6) eine:Bestimmungsmethode für $\mathrm{H}-\mathrm{TSH}$ unter Einsatz von markiertem bovinen TSH (B-TSH) und B-TSH-Antikörpern. Sie postulierten damit im Gegensatz zu UTIGER und Mitarbeitern (4) eine Kreuzimmunität zwischen H-TSH und B-TSH. FREYCHET und Mitarbeitern (7) wiederum gelang die Bestimmung von $\mathrm{H}$-TSH mit Antikörpern gegen Schweine-TSH (P-TSH). Ferner fanden sie bei Verwendung eines speziellen P-TSHAntiserums eine - allerdings unvollständige - Verdrängung von markiertem B-TSH durch H-TSH. Im „,homologen“ H-TSH-System (markiertes H-TSH, HTSH-Antikörper) konnten auch hohe Dosen von B-TSH und P-TSH markiertes H-TSH nicht aus seiner spezifischen Bindung verdrängen. Auch ODELL und Mitarbeiter (8) erzielten in diesem System keine Hemmung durch TSH von Rindern, Vögeln oder Mäusen. Es ist 
also noch nicht endgültig geklärt, ob und inwieweit sich "heterologe" Systeme zum radioimmunchemischen Nachweis von H-TSH eignen.

Obwohl zur Zeit zwei homologe H-TSH-Systeme hierfür zur Verfügung stehen und erste Erfahrungen mehrerer Arbeitsgruppen mit einem von ihnen vorliegen, bereitet der Vergleich der Ergebnisse wegen der noch nicht ausreichend standardisierten Technik und der noch nicht genügend geklärten Frage ihrer Sensibilität Schwierigkeiten. Im nachfolgenden Bericht wollen wir auf Grund unserer Erfahrungen mit der radioimmunchemischen Bestimmung von H-TSH zu folgenden Punkten Stellung nehmen:

1. Methodik (heterologes und homologes System),

2. Eignung eines heterologen Systems (B-TSH),

3. Vergleich verschiedener H-TSH- und H-LH-Präparationen im Hinblick auf ihre Kreuzimmunität unter Verwendung der zur Verfügung stehenden Antikörper,

4. Ergebnisse der Bestimmung von H-TSH im menschlichen Plasma.

\section{Material und Methoden}

\section{Hormonpräparationen zur Markierung}

B-TSH: Gereinigte Thytropar (s. u.) der Armour Pharmaceutical Comp., USA.

H-TSH: Präpatationen des National Institute of Health (NIH), Bethesda, USA, sowie der Firma Calbiochem, Los Angeles, USA (Lot No. 079506).

H-LH: Präparationen des NIH (LER 960) sowie der Firma Calbiochem (Lot No. 079503).

Standard-Präparationen des National Institute for Medical Research (NIMR), London, England:

Thyrotropin, Bovine, for Bioassay,

The Human Thyrotropin Research Standard A,

Human Menopausal Gonadotrophins, Urinary, for Bioassay (2-IRP-HMG),

Growth Hormone, Human, for Immunoassay.

\section{Antikörper}

Gegen Hormone:

B-TSH: Gewinnung s. u.

H-TSH: Präparationen des NIH (Kaninchen) sowie der Firma Calbiochem (Lot No. 079507; Kaninchen).

H-LH: Präparationen des NIH (HCG-Antiserum; Kaninchen) sowie der Firma Calbiochem (Lot No. 079504; Kaninchen). Gegen $\boldsymbol{\gamma}$-Globuline (Doppelantikörper):

Präparationen der Firma Sylvana Comp., Millburn, USA:

Anti-Kaninchen-Globulinserum (Kat. Nr. 20-264; Schaf), Anti-Meerschweinchen-Globulinserum (Kat. Nr. 20-238; Kaninchen).

HCG der Firma Organon (2580 IU/mg).

\section{Radiojod zur Einveißmarkierung}

131 J: Firma Hoechst, Deutschland (J 1006, spezifische Aktivität $20-25 \mathrm{C} / \mathrm{mg}$ ),

Radiochemical Centre, Amersham, England (IBS 3; spezifische Aktivität $6-20 \mathrm{C} / \mathrm{mg}$ ).

${ }^{125} \mathrm{~J}$ : Radiochemical Centre, Amersham, England (IMS 3; spezifische Aktivität 4-12 C/mg),

NEN Chemicals GmbH, Boston, USA (keine Angaben zur spezifischen Aktivität).

\section{Puffer}

I $-0,5 \mathrm{M}$ Phosphatpuffer $\mathrm{pH} 7,5$.

II $-0,01 \mathrm{M}$ Phosphatpuffer $+0,15 \mathrm{M} \mathrm{NaCl} \mathrm{pH} 7,8$.

III - Puffer II + 0,5\% Rinder-Serumalbumin der Firma Behringwerke, Deutschland.

Alle Pufferlösungen wurden wöchentlich frisch angesetzt.

\section{Aufbewabrung}

In Puffern verdünnte und in kleine 'Portionen aufgeteilte Standards, hochgereinigte Hormonpräparationen und spezifische Antiseren bei $-80^{\circ}$; Antigammaglobulin-Seren sowie PatientenPlasmen bei $-20^{\circ}$. Mehrfaches Auftauen und Wiedereinfrieren wurde vermieden.

\section{Reinigung von kommerziellem $B-T S H$}

B-TSH (Thytropar) wurde über eine CM 52-Säule (Whatman) durch Gradientenelution gereinigt (0,01 Phosphatpuffer pH 6,25/ $1 \mathrm{M} \mathrm{NaCl}$; modifiziert nach Condlifre und Mitarbeitern (9)), wobei sich zwei proteinhaltige Fraktionen trennen ließen. Die biologische TSH-Aktivität (MCKENZrE-Test (10)) der zweiten Fraktion betrug pro Gewichtseinheit Protein etwa das 5 fache der ersten. Nach Dialysieren und Lyophilisieren wurde Fraktion II durch Celluloseacetatfolien- und Diskelektrophorese auf ihre Homogenität geprüft. Mit beiden Verfahren konnte nur eine Eiweißbande dargestellt werden, im Gegensatz zu vier oder fünf Banden bei elektrophoretischer Trennung von ungereinigtem Thytropar. Die lyophilisierte Fraktion II ließ sich vollständig in Phosphatpuffer pH 7,8 lösen.

\section{Gewinnung von Antikörpern gegen B-TSH}

Fünf weibliche, etwa $400 \mathrm{~g}$ schwere, genetisch differente Meerschweinchen wurden in 14tägigen Abständen durch wiederholte Injektionen einer wäBr. Lösung von je 1,5 IU B-TSH (Thytropar) zusammen mit Freund'schem kompletten Adjuvans (Wasser-inOl-Emulsion) in die Pfoten und s. c. immunisiert.

Nach sieben Injektionen wurde in oberflächlicher Äthernarkose durch Herzpunktion Blut entnommen und das abgetrennte Serum, mit albuminhaltigem Phosphatpuffer verdünnt, in kleineren Portionen eingefroren.

Bei allen immunisierten Meerschweinchen konnten Antikörper gegen B-TSH immunelektrophoretisch, durch Neutralisation der biologischen Aktivität im MCKENZIE-Test sowie durch Bindung von markiertem B-TSH an Serumverdünnungen nachgewiesen werden (11).

\section{Markierung der Hormonpräparationen}

B-TSH (gereinigtes Thytropar), H-TSH (NIH bzw. Calbiochem) und L-LH (LER 960) wurden nach GREENwOOD und HuNTER (12) in folgender Weise markiert:

In ein kleines, chemisch reines Plastik-Röhrchen wurden bei Raumtemperatur nacheinander unter ständigem Schütteln mit Konstriktions-Pipetten eingebracht:

$2 \mathrm{mC}^{131} \mathrm{~J}$ oder ${ }^{125} \mathrm{~J}(10-50 \mu l)$

Puffer I $(20-50 \mu l)$.

B-TSH, H-TSH oder H-LH, $5 \mu$ gelöst in Puffer II $(10-25 \mu l)$ Chloramin T, $25 \mu \mathrm{g}$, gelöst in Puffer II $(10 \mu \mathrm{l})$.

Nach einer Reaktionszeit von etwa $30 \mathrm{Sek}$. Zugabe von $62,5 \mu \mathrm{g}$ Natriumsulfit, gelöst in $100 \mu l$ Puffer II. Die Trennung des markierten Hormons von überschüssigem Jodid und Oxydationsbzw. Reduktionsmittel erfolgte unmittelbar danach über eine kleine Sephadex-Säule (Sephadex-G 50 oder G $750,5 \times 10 \mathrm{~cm}$; äquilibriert mit Puffer II, abgesättigt mit $1 \mathrm{ml} 2$ proz. RinderSerumalbumin-Lösung; Elution mit Puffer II) in Fraktionen zu $0,5 \mathrm{ml}$, gesammelt über $100 \mu l$ 2proz. Rinder-SerumalbuminLösung. Radioaktivitätsmessung der Fraktionen unter gleichbleibenden geometrischen Bedingungen über einem Bohrlochdetektor mit Impulshöhenanalysator. Prüfung der Gipfelfraktionen des ersten „Proteingipfels“ auf radioaktive Degradationsprodukte durch Papierelektrophorese (Whatman 3 MM, Veronal-Puffer, 
$\mathrm{pH} 8,6,0,5 \mathrm{~mA} / \mathrm{cm}$ Papierbreite, 1 Std. Laufzeit bei $+4^{\circ}$ (13)). Hierbei bleibt das markierte Hormon an der Auftragsstelle (Kathode) liegen, markierte Degradationsprodukte wandern nur wenig, freies Radiojodid findet sich weit anodenwärts. Aufbewahrung der reinsten Fraktion bei $+4^{\circ}$ nach Verdünnung mit Puffer III. In orientierenden Versuchen erfolgte eine Nachreinigung von $0,5 \mathrm{ml}$ der Gipfelfraktion nach 10 minütiger Inkubation mit $0,5 \mathrm{ml}$ Hypophysektomierten-Plasma bei $+4^{\circ}$ (Sephadex-G 100-Säule, $0,9 \times 45 \mathrm{~cm}$; Äquilibrierung und Elution mit Puffer II). Ursprünglich bestimmten wir die spezifische Aktivität des markierten Hormons durch Fällung einer verdünnten Probe des Markierungsansatzes vor Säulentrennung mit 10proz. Trichloressigsäure und anschließendem Vergleich der Radioaktivität der Fällung mit einem Radiojodstandard bekannter Aktivität. $\mathrm{Da}$ nach Vorversuchen immer die gleichen Radioaktivitäts- und Hormonmengen $(2 \mathrm{mC}, 5 \mu \mathrm{g}$ ) eingesetzt wurden, konnte die Qualität der Markierungen auch über den Prozentsatz der eiweißgebundenen Radioaktivität (,Trichlotessigsäure-Faktor") direkt miteinander verglichen werden.

Mit ${ }^{131} \mathrm{~J}$-markierten Hormonpräparaten wurde nur in den ersten 3 Tagen, mit ${ }^{125} \mathrm{~J}$-markierten nicht länger als eine Woche nach Markierung gearbeitet.

\section{Ansatz und Durchfubrung der radioimmunchemischen Reaktion und Trennung der Reaktionsprodukte}

Bei Durchführung der radioimmunchemischen Reaktion folgten wir, mit einigen Modifikationen, den Angaben von Odell (14), des NIH (15) und Lemarchand-Beraud (6). Alle Reagenzien, gelöst bzw. verdünnt in Puffer III, wurden mit automatischen Pipetten (Mikropipetten Marburg, Firma Eppendorf) in verschließbare konische Plastikröhrchen (Reaktionsgefäße 3810, Firma Eppendorf) pipettiert (Pipettierfehler $\pm 1 \%$ ). Die Zugabe von EDTA $(0,01 \mathrm{M})$ sollte, ebenso wie der Einsatz von Plasma anstatt Serum, durch Inaktivierung von Komplement bzw. Komplement-Faktoren eine eventuelle Hemmung der Doppelantikörper-Reaktion verhindern (16). Zum H-TSH-System wurden pro Ansatz 10 IU HCG hinzugefügt, um gonadotropinbindende Valenzen am H-TSH-Antikörper abzusättigen. Bei einigen Versuchen wurden den Ansätzen der Standardkurve entsprechende Mengen Hypophysektomierten-Plasma zugegeben, um hinsichtlich der Eiweißkonzentration vergleichbare Bedingungen mit den Plasmaansätzen zu schaffen.

In Vorversuchen wurde die Verdünnung des Antikörpers ermittelt, bei der in Abwesenheit unmarkierten Antigens 40-60\% des radioaktiven Hormons gebunden wurden. Nach Errechnung der spezifischen Aktivität des markierten Hormons wurden $0,05-0,15 \mathrm{ng}$ desselben, entsprechend $1 \times 10^{4}$ bis $2 \times 10^{4} \mathrm{Imp} . /$ Min. in unserer Meßanordnung, pro Ansatz eingegeben (Tab. 1). Nach 5 tägiger Äquilibrierung bei $+4^{\circ}$ wurde das antikörpergebundene vom freien Hormon durch Zugabe eines gegen den Antikörper gerichteten, präzipitierenden „Doppelantikörpers“ (Antiglobulinserum) im Úberschuß abgetrennt (17). Nach anfänglichen Versuchen mit Membranfiltern (Millipore HAWE
$02500,25 \mathrm{ea}, \mathrm{HA} \quad 0,45 \mu \mathrm{m}$; Sartorius SM 11306) sowie Ionenaustauschern (Amberlite) isolierten wir schließlich den Doppelantikörperkomplex durch Zentrifugieren $(15000 \mathrm{~g}, 3 \mathrm{Min}$.) und bestimmten die Radiojodaktivität des Präzipitates direkt im Reaktionsgefäß nach Absaugen des Uberstandes in einem automatischen Probenwechsler mit Impulshöhenanalyse (Meßfehler $<1,5 \%)$. Um ein gut sichtbares Präzipitat zu crhalten, wurden gleichzeitig mit dem Doppelantikörper $20 \mu l$ 1:40 verdünntes Serum von unbehandelten Meerschweinchen bzw. Kaninchen in die Ansätze pipettiert.

Neben einer Standard-Kurve (logarithmische Verdünnung einer TSH-Standard-Präparation) wurden in jeder Versuchsserie folgende Kontrollen mitbestimmt:

A. Eine Probe, welche nur Antikörper und markiertes Hormon enthielt, zur Kennzeichnung des unteren Grenzwertes der Bestimmungsmethode und der Antikörper-Bindung des markierten Hormons (Angabe in \% der Probe C);

B. eine Probe, die anstelle des spezifischen Antikörpers das Serum eines nicht immunisierten Meerschweinchens bzw. Kaninchens in gleicher Verdünnung enthielt, zur Feststellung der unspezifischen Bindung des markierten Hormons oder radioaktiver Degradationsprodukte an Eiweißkörper im Versuchsansatz. Die Impulsrate dieser Probe wurde später von allen Einzelwerten subtrahiert;

C. eine Probe, die lediglich markiertes Hormon enthielt und als Hundertprozent-Wert eingesetzt wurde;

D. eine Probe mit Hypophysektomierten-Plasma;

E. eine Probe mittleren TSH-Gehaltes (Pool-Plasma), um einen Vergleich der Ergebnisse verschiedener Versuchsserien (Reproduzierbarkeit) zu ermöglichen.

Sämtliche Proben (Standard-Kurve, Kontrollen, Patienten-Plasma) wurden 3 fach angesctzt.

\section{Statistische Berechnungen}

Für eine Reihe von Standard-Kurven wurden im halblogarithmischen System Regressionsgeraden berechnet. Als Maßstab für ihre Qualität dienten der Präzisionsindex $\lambda$ (18), definiert als Quotient aus der Standardabweichung (s) (Streuung des Einzelwertes um die Regressionsgerade) und dem Regressionskoeffizienten (b), sowie der Korrelationskoeffizient ( $r$ ).

\section{Ergebnisse}

Das pH-Optimum der Oxydationsreaktion von Radiojodid mit Chloramin T liegt bei pH 7,5 (19). Kommerzielles Radiojod zur Eiweißmarkierung wird in wechselnden Volumina $\mathrm{NaOH}$ (etwa $0,01 \mathrm{~N}$ ) mit einem $\mathrm{pH}$ von 8-10 geliefert. Die eingesetzte Menge des Puffer I muß deshalb jeweils variiert werden. $\mathrm{Da}$ wegen des kleinen Reaktionsvolumens (um $50 \mu l$ ) direkte $\mathrm{pH}$ -

Tab. 1

Reaktionsansätze des B-TSH- und H-TSH-Systems

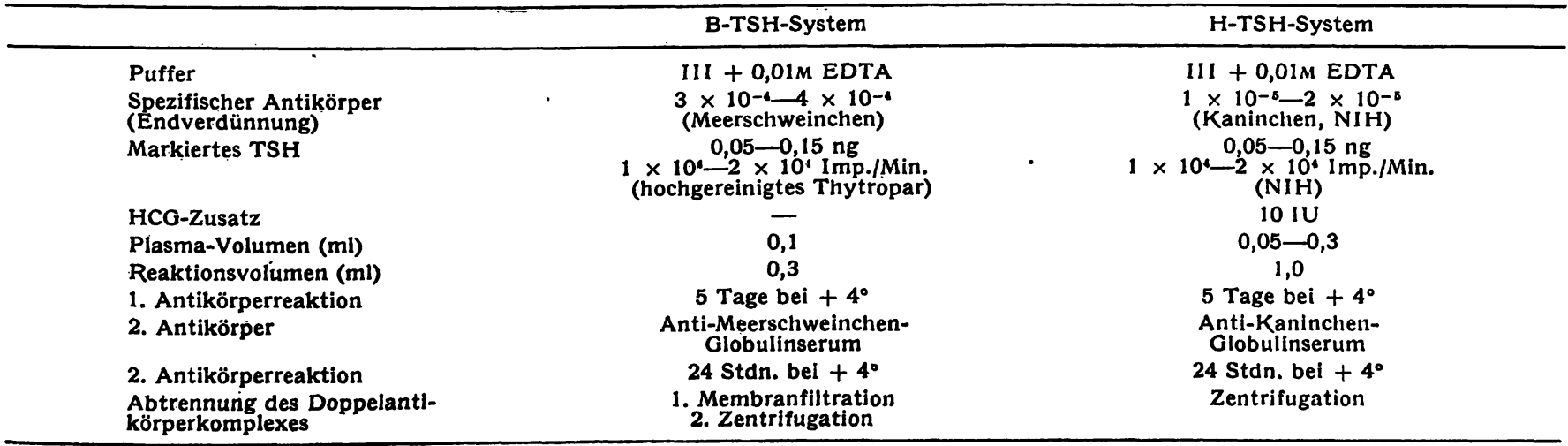


Tab. 2

Einfluß des Phosphatpuffer-Volumens ( $\mathrm{pH} 7,5 ; 0,5 \mathrm{M}$ ) im MarkierungsEnsatz auf die spezifische Radioaktivität (Trichloressigsäure-Faktor) des Hormons

\begin{tabular}{lccc}
\hline & & Markierung I & Markierung II \\
\hline Puffer I & $(\mu l)$ & 25 & 50 \\
isiJ & $(\mu l)$ & 30 & 30 \\
H-TSH & $(\mu l)$ & 10 & 10 \\
Chloramin T & $(\mu l)$ & 10 & 10 \\
Natriumdisulfit & $(\mu l)$ & 100 & 100 \\
Trichloressigsäure-Faktor $(\%)$ & 46 & 71 \\
\hline
\end{tabular}

Messungen schwer möglich sind, setzten wir Puffer und Radioaktivität immer im Volumenverhältnis 5:3 ein. Das Beispiel in Tabelle 2 zeigt unter Verwendung derselben ${ }^{131} \mathrm{~J}$-Charge die Wichtigkeit dieses Vorgehens. Bei der papierelektrophoretischen Überprüfung der Fraktionen des ersten „Proteingipfels“ nach säulenchromatographischer Auftrennung konnten in der radioaktivsten Probe bei guter Markierung (Trichloressigsäure-Faktor $>70 \%$ ) weniger als $5 \%$ radioaktive Degradationsprodukte bzw. freies Radiojodid nachgewiesen werden. Entsprechend waren 97\% der Radioaktivität dieser Probe mit 10proz. Trichloressigsäure fällbar, d. h. eiweißgebunden.

Eine zusätzliche Reinigung der Gipfelfraktion (H-TSH) über Sephadex-G 100, wie von MelanI und Mitarbeitern (20) für Wachstumshormon vorgeschlagen, brachte keine entscheidende Verbesserung der Statistik der Standardkurven (vor Reinigung: $s=52$ Imp./Min.; $b=$ 196 Imp./Min.; $\hat{\lambda}=0,26$; $r=0,987$; nach Reinigung: $\mathrm{s}=42 \mathrm{Imp} . / \mathrm{Min} . ; \mathrm{b}=200 \mathrm{Imp} \cdot /$ Min. $\lambda=0,21 ; \mathrm{r}=$ 0,992).

Trotz weitgehender Standardisierung des Markierungsprozesses wurden erhebliche Schwankungen des Trichloressigsäure-Faktors als Parameter der spezifischen Aktivität beobachtet (Tabelle 3). Bei Einsatz von $2 \mathrm{mC}$ ${ }^{131} \mathrm{~J}$ und $5 \mu \mathrm{g}$ Hormon entspricht ein Trichloressigsäure-

Tab. 3

Trichloressigsäure-Faktor als Ausdruck der spezifischen Aktivität bei ${ }^{131} \mathrm{~J}$-Markierungen (J 1006 Firma Hoechst, $2 \mathrm{mC}$ ) von H-TSH (NIH, $5 \mu \mathrm{g})$

\begin{tabular}{cc}
\hline Datum der Markierung & Trichloressigsäure-Faktor \\
\hline 21.1 .1970 & 65 \\
4.2 .1970 & 43 \\
2.4 .1970 & 67 \\
14. 4. 1970 & 64 \\
29. 4.1970 & 65 \\
5. 5.1970 & 90 \\
9.6 .1970 & 71 \\
16.6 .1970 & 82 \\
23.6 .1970 & 49 \\
1.7 .1970 & 77 \\
7.7 .1970 & 77 \\
21.7 .1970 & 76 \\
28.7 .1970 & 73 \\
4.8 .1970 & 55 \\
& 50
\end{tabular}

Faktor von $70 \%$ etwa einer spezifischen Aktivität von $280 \mathrm{mC} / \mathrm{mg}$. Mit ${ }^{125} \mathrm{~J}$ erreichten wir bei B-TSH spezifische Aktivitäten um $110 \mathrm{mC} / \mathrm{mg}$ und bei $\mathrm{H}$-TSH um $140 \mathrm{mC} / \mathrm{mg}$, mit ${ }^{131} \mathrm{~J}$ um $150 \mathrm{mC} / \mathrm{mg}$ bzw. um $280 \mathrm{mC} / \mathrm{mg}$.

\section{Reaktionsansatz und Trennung der Reaktionsprodukte}

Je nach Verdünnung des Antikörpers (s. Tab. 1) wurden in Abwesenheit von unmarkiertem Antigen 40-60\% des eingesetzten radioaktiven Hormons gebunden (Kon- trolle $\mathrm{A}$ in \% von Kontrolle C). Dabei war die Bindungsfähigkeit des Antikörpers bei gleicher Verdünnung für ${ }^{125} \mathrm{~J}$-markiertes H-TSH oder B-TSH um $10-20 \%$ geringer als für ${ }^{131} \mathrm{~J}$-markiertes TSH. Die „unspezifische Bindung“ des ${ }^{131} \mathrm{~J}$-markierten H-TSH bzw. radioaktiver Degradationsprodukte an Eiweißkörper betrug 2-5\% (Kontrolle B in \% von Kontrolle A). Dieser Prozentsatz zeigte keine Abhängigkeit vom Trichloressigsäure-Faktor. Er erhöhte sich maximal um $1,5 \%$ bei Verwendung der ${ }^{131} \mathrm{~J}-\mathrm{H}$-TSH-Markierung innerhalb der ersten 3 Tage.

Wurde den Ansätzen der Standardkurve (H-TSH) $0,3 \mathrm{ml}$ Hypophysektomierten-Plasma zugefügt, lag die Antikörper-Bindung von ${ }^{131} \mathrm{~J}-\mathrm{H}-\mathrm{TSH}$ gegenüber den nur mit Puffer III angesetzten Standardkurven um 10 bis $15 \%$ niedriger. Folglich wurden auch niedrigere TSH-Werte in Patienten-Plasmen von diesen Kurven abgelesen. Ein statistischer Vergleich. der Standardkurven ergab jedoch weder für ihre Regressionskoeffizienten noch für ihre Varianz signifikante Unterschiede (Puffer: $s=52$ Imp./Min.; $b=196$ Imp./Min.; $\lambda=$ 0,26; $\mathbf{r}=0,987$. Plasma: $\mathbf{s}=38$ Imp./Min.; $b=199$ Imp./Min.; $\lambda=0,19 ; \mathrm{r}=0,993$ ). Auch der Prozentsatz der unspezifischen Bindung (Kontrolle B) war bei beiden Verfahren identisch.

Durch 24stdg. Inkubation von Antikörpern und Standardverdünnung bzw. Patienten-Plasma vor Zugabe des markierten TSH (nicht äquilibriertes System; Methode C nach Hales und Randle (17)) ließ sich die Empfindlichkeit des H-TSH-Systems nicht steigern. Wurde das nicht äquilibrierte System vor Zugabe von ${ }^{131} \mathrm{~J}$-markiertem H-TSH $6 \mathrm{Stdn}$. und dann noch bis zum Hinzufügen des Doppelantikörpers weitere $20 \mathrm{Stdn}$. bei $+37^{\circ}$ inkubiert (21), nahmen die Antikörper-Bindung von markiertem TSH sowie die Steilheit der Standardkurve deutlich $a b$, die unspezifische Bindung (Kontrolle B) und die Streuung der Einzelwerte hingegen zu. Orientierende Versuche mit der Adsorption von B-TSHAntikörpern an unlösliche Polymere (TetrafluoräthylenScheibchen (22)) wurden wegen stark streuender Einzelwerte und des relativ hohen Antikörper-Verbrauches verlassen.

Eine ausreichende Trennung des antikörpergebundenen vom freien Hormon durch Amberlite (bei niedermolekularen Proteohormonen exfolgreich angewandt) gelang im B-TSH-System nicht. Die Isolierung des Doppelantikörper-Komplexes erfolgte durch Zentrifugation, nachdem bei der Membranfiltration eine größere Streuung der Einzelwerte, abhängig von der jeweils benutzten Membranfilter-Charge, beobachtet wurde und dieses Verfahren auch zeitraubender ist.

\section{Spezifität}

Unsere Untersuchungen zur Spezifität des B-TSH- und H-TSH-Systems führten zu folgenden Ergebnissen:

1. Im B-TSH-System (B-TSH markiert mit ${ }^{125} \mathrm{~J}$ und ${ }^{131} \mathrm{~J}, \mathrm{~B}-\mathrm{TSH}$-Antikörper) erzielten wir reproduzierbare Standardkurven $(\lambda=0,19 ; \mathrm{r}=0,991)$ über 
einen Bereich von 5,0-160 $\mu \mathrm{IU}$ (IS Thyrotrophin, Bovine for Bioassay). Beim Einsatz von H-TSH als Standard (The Human Thyrotropin Research Standard A) und aller B-TSH-Antiseren konnte auch in höheren Dosisbereichen (bis $500 \mu \mathrm{U}$ H-TSH) keine ausreichende Verdrängung von markiertem B-TSH beobachtet werden (s. auch (11)). In keinem Fall konnten wir bei Verwendung des B-TSH-Systems TSH-Spiegel im Plasma primär hypothyreoter Patienten bestimmen.

2. Im H-TSH-System (H-TSH markiert mit ${ }^{131} \mathrm{~J}, \mathrm{H}-$ TSH-Antikörper; beides Präparationen des NIH und in orientierenden Versuchen der Firma Calbiochem) ergaben sich unter Verwendung des Human Thyrotropin Research Standard A, der hochreinen, üblicherweise zur Markierung benutzten H-TSH-Präparationen des NIH $(10,4 \mathrm{IU} / \mathrm{mg})$ und der Firma Calbiochem (5-7 IU/mg), sowie von Plasma-Verdünnungen primär hypothyreoter Patienten vergleichbare Standardkurven über einen Bereich von 0,78 bis $100 \mu \mathrm{U}$ (Human Thyrotropin Research Standard A) bzw. 0,39-50 ng (NIH, Calbiochem)), wenngleich ihre Steilheit Unterschiede aufwies (Abb. 1).

Ebenso wie ODELL und Mitarbeiter (8) konnten wir weder eine Bindung von markiertem B-TSH an H-TSH-

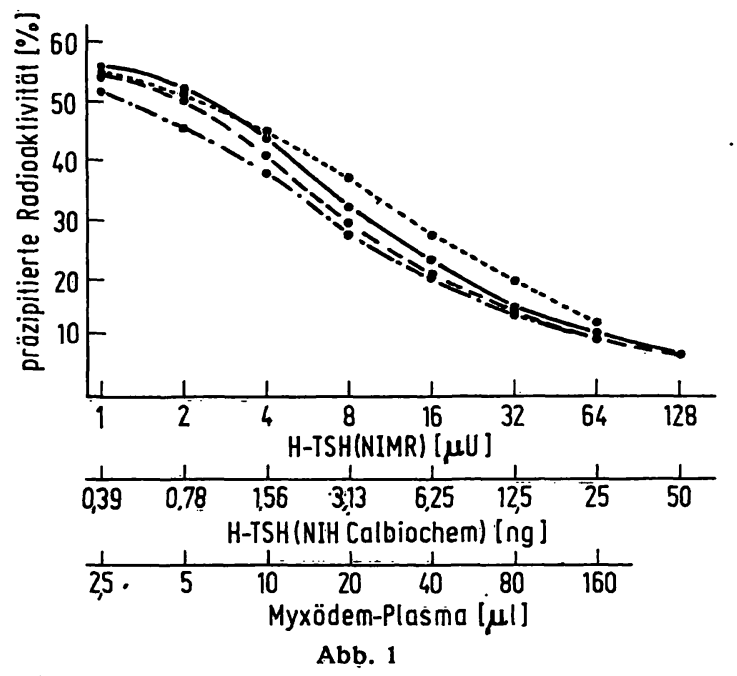

Standardkurven verschịedener H-TSH-Präparationen im H-TSHSystem (NIH)

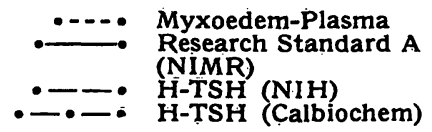

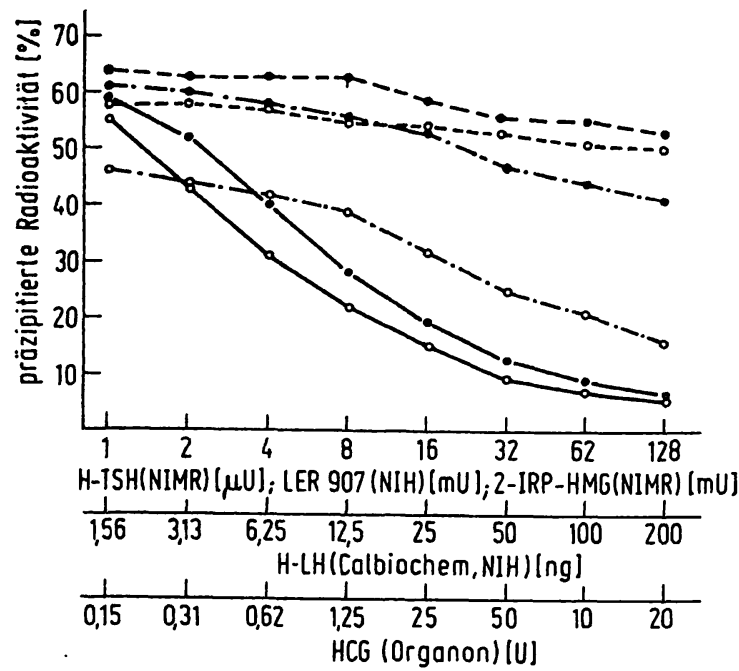

Abb. 2

Das Verhalten verschiedener Gonadotropin-Präparationen im H-TSHSystem (NIH)

$\because-0$ H-TSH (NIMR)

$x \longrightarrow x \quad$ LER 907 (NIH)

2-IRP-HMG (NIMR)

$\because-\bullet-\bullet \quad H-L H$ (Calbiochem)

$x-\cdot-x \quad \mathrm{H}-\mathrm{LH}(\mathrm{NIH})$

$x \cdots x$ HCG (Organon)

Antikörper (NIH) noch eine Verdrängung von markiertem $\mathrm{H}$-TSH vom spezifischen Antikörper durch B-TSH beobachten.

Zur Frage der Kreuzimmunität von H-TSH und H-LH wurden im H-TSH-System (markiertes H-TSH und Antiserum des NIH, ohne HCG-Zusatz) das Verhalten von vier Gonadotropin-Präparationen getestet (Abb. 2, Tab. 4). LER 907, einheitsmäßig umgerechnet auf 2IRP-HMG, verdrängt - entsprechend seinem hohen H-TSH-Gehalt $(213 \mu \mathrm{U}$ pro $128 \mathrm{mIU} \mathrm{LH})-$ in ausgeprägtem Maße markiertes $\mathrm{H}$-TSH vom spezifischen Antikörper. H-LH (Calbiochem) ist geringer durch $\mathrm{H}-\mathrm{TSH}$ verunreinigt $(6 \mu \mathrm{U}$ TSH pro $100 \mathrm{ng} \mathrm{LH}):$ Es resultiert lediglich eine flache Kurve im H-TSH-System. Eine andere H-LH-Präparation (NIH) enthält vergleichsweise mehr H-TSH ( $40 \mu \mathrm{U}$ TSH pro $100 \mathrm{ng} \mathrm{LH}$ ), was sich auch deutlich im Kurvenverlauf zeigt. HCG und 2-IRP-HMG enthalten kein TSH; ihre Kreuzimmunität zum H-TSH-Antikörper (NIH) ist gering.

Beim Einsatz von Wachstumshormon (NIMR; bis $10 \mathrm{ng}$ ) wurde keine Kreuzreaktion im H-TSH-System (NIH) beobachtet:

Tab. 4

TSH- und LH-Gehalt verschiedener Hormonpräparationen $(23,24)$

\begin{tabular}{|c|c|c|}
\hline & $\begin{array}{c}\text { TSH-Gehalt in IU } \\
\text { (biologisch getestet; } \\
\text { bezogen auf IRP Thyro- } \\
\text { trophin, Bovine, for } \\
\text { Bioassay; I IU }=13,5 \mathrm{mg} \text { ) }\end{array}$ & $\begin{array}{c}\text { LH-Gehalt in IU } \\
\text { (biologisch getestet; } \\
\text { bezogen auf 2-IRP-HMG; } \\
40 \text { IU/Ampulle) }\end{array}$ \\
\hline $\begin{array}{l}\text { The Human Thyrotropin } \\
\text { Research Standard A } \\
\text { (NIMR; vorläufige Angabe } \\
\text { der TSH-Aktivität in U) }\end{array}$ & $\begin{array}{c}1,15 / \mathrm{mg} \\
(0,05 / \text { Ampulle })\end{array}$ & $\begin{array}{l}6060 / \mathrm{mg} \\
\text { (200/Ampulle) }\end{array}$ \\
\hline $\begin{array}{l}\text { H-TSH (NIH) } \\
\text { H-TSH (Calbiochem) } \\
\text { H-LH (LER 960; NIH) } \\
\text { H-LH (Calblochem) } \\
\text { Hypophýsenextrakt } \\
\text { (LER 907; NIH) }\end{array}$ & $\begin{array}{c}10,40 / \mathrm{mg} \\
5,00-7,00 / \mathrm{mg} \\
0,40 / \mathrm{mg} \\
0,06 / \mathrm{mg} \\
0,08 / \mathrm{mg}\end{array}$ & $\begin{array}{r}0,165 / \mathrm{mg} \\
100 / \mathrm{mg} \\
932 / \mathrm{mg} \\
2000 / \mathrm{mg} \\
48 / \mathrm{mg}\end{array}$ \\
\hline
\end{tabular}


Im radioimmunchemischen $\mathrm{H}$-LH-System (markiertes H-LH (LER 960) des NIH; H-LH-Antikörper der Firma Calbiochem (Endverdünnung 0,8 $\times 10^{5}$ )) reagieren sowohl Human Thyrotropin Research Standard A mit hohem LH-Anteil (512 mIU LH pro $128 \mu \mathrm{U}$ TSH) als auch H-TSH (NIH) mit sehr geringem LH-Gehalt (0,0165 mIU LH pro $100 \mathrm{ng}$ TSH) stark mit dem LHAntikörper. Das gleiche ist zu beobachten, wenn man anstelle des LH-Antikörpers (Calbiochem) einen HCGAntikörper (NIH; Endverdünnung $1 \times 10^{5}$ ) verwendet. Dieses Verhalten spricht für eine Kreuzimmunität zwischen $\mathrm{H}$-LH und H-TSH beim Einsatz der beiden genannten Antiseren (Abb. 3).

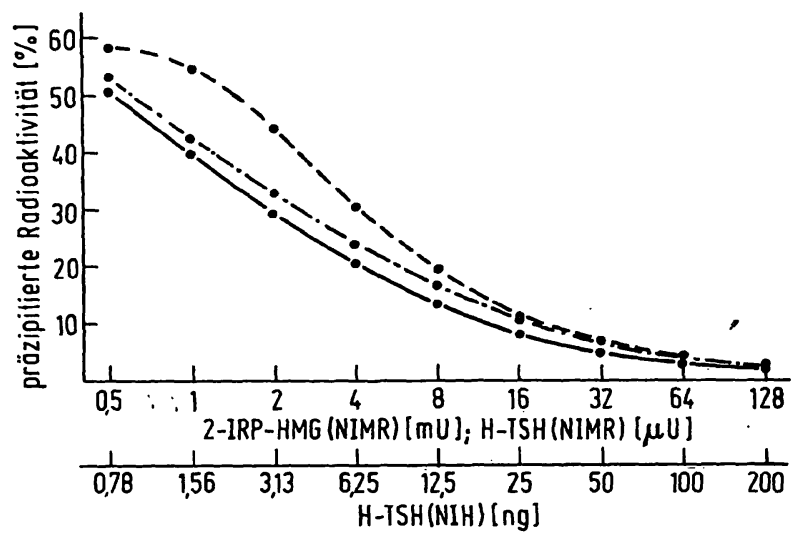

Abb. 3

Das Verhalten von zwei H-TSH-Präparationen im H-LH-System (H-LH: NIH; H-LH-Antiserum: Calbiochem)

- - - Research Standard A (NIMR)

-..... H-TSH (NIH)

•— LER 907 (NIH)

\section{Empfindlicbkeit, Genauigkeit, Reproduzierbarkeit}

Die Empfindlichkeit des H-TSH-Systems lag bei $0,5 \mu \mathrm{U}$ Human Thyrotropin Research Standard A bzw. bei $1,5-2 \mu \mathrm{U} / \mathrm{m} l$ Plasma.

Die Genauigkeit, bei wiederholter Bestimmung der gleichen Probe im selben Versuchsansatz (Variationskoeffizient), betrug $\pm 5 \%$.

Die Reproduzierbarkeit, bei wiederholter Bestimmung der gleichen Probe in verschiedenen Versuchs-

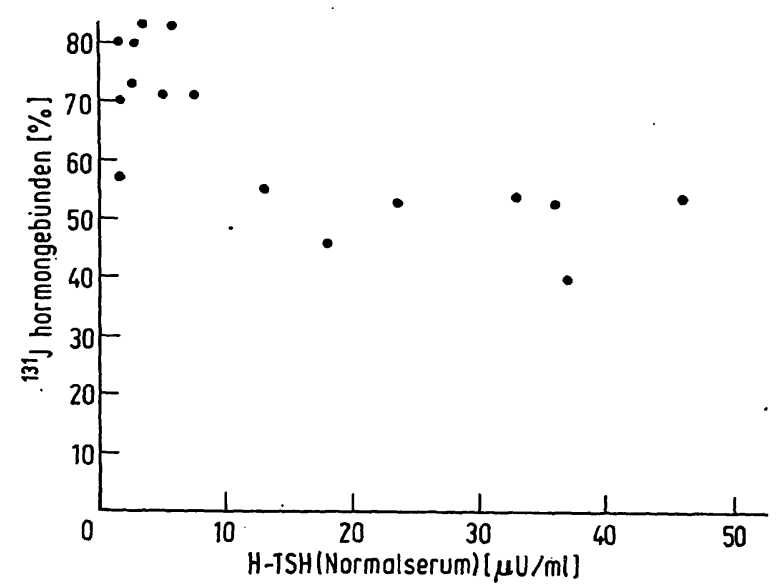

Abb. 4

Ergebnis wiederholter Bestimmungen des TSH-Gehaltes der gleichen Plasmaprobe unter Verwendung von ${ }^{131} \mathrm{~J}-\mathrm{H}-\mathrm{TSH}(\mathrm{NIH})$ verschiedener spezifischer Aktivität (,TCA"-Faktor) ansätzen unter Verwendung von ${ }^{131} \mathrm{~J}-\mathrm{H}-\mathrm{TSH}$-Markierungen vergleichbarer spezifischer Aktivität, betrug in den niederen Konzentrationsbereichen (um $5 \mu \mathrm{U} / \mathrm{m} /$ ) $\pm 20 \%$ und in den höheren (um $150 \mu \mathrm{U} / \mathrm{m}$ ) $\pm 10 \%$. Bei niedriger spezifischer Aktivität des markierten HTSH wurden höhere TSH-Spiegel in der Probe gemessen (Abb. 4).

\section{Untersucbungen mit menschlichem Plasma (H-TSH-System)}

Normalkollektiv: 32 schilddrüsengesunde Personen (15 Frauen, 17 Männer) überwiegend im Alter zwischen 20 und 30 Jahren.

Die Plasmen wurden in einer Versuchsserie bestimmt. Nach Abzug des Wertes für ein HypophysektomiertenPlasma lagen in $60 \%$ die Ergebnisse unter der methodischen Erfaßbarkeit. Die reștlichen Normalpersonen hatten TSH-Plasmaspiegel bis $6 \mu \mathrm{U} / \mathrm{m} l$.

Hyperthyreote Patienten: Von 10 Patienten mit unbehandelter Schilddrüsenüberfunktion wiesen nur drei nach Abzug des Wertes für ein Hypophysektomierten-Plasma einen meßbaren TSH-Plasmaspiegel auf, ebenfalls bis maximal $6 \mu \mathrm{U} / \mathrm{m} l$.

Hypothyreote Patienten: Bei 25 Patienten mit primärer Schilddrüsenunterfunktion aller Schweregrade und verschiedener Ätiologie (Thyreoiditis HASHrmoto, Jod-Fehlverwertung, Struma maligna nach Thyreoidektomie, Hypothyreose nach Strumektomie, ${ }^{131} \mathrm{~J}$-Therapie, Überdosierung von Thyreostatika) fanden sich TSHSpiegel von $12-600 \mu \mathrm{U} / \mathrm{m} l$ Plasma.

\section{Diskussion}

Zur Durchführung radioimmunchemischer Hormonbestimmungen sind folgende Voraussetzungen erforderlich:

1. Eine hochgereinigte Hormonpräparation, schonend bis zu einer ausreichenden hohen spezifischen Aktivität markiert;

2. ein spezifischer oder kreuzimmuner Antikörper, der noch in starker Verdünnung eine bevorzugte Affinität zum Antigen (markierte Hormonpräparation, Standard) zeigt;

3. eine Standardpräparation, die gegenüber dem Antikörper das gleiche Verhalten zeigt wie das markierte Hormon.

Neben der Verwendung eines potenten Antikörpers ist die Qualität des markierten Hormons für die Empfindlichkeit einer tadioimmunologischen Methode und die Reproduzierbarkeit der Ergebnisse von entscheidender Bedeutung. Einer der Parameter für die Qualität der Markierung ist die spezifische Aktivität, deren Berechnung nur approximativ möglich ist. Aus Vergleichsgründen sollte der Berechnungsmodus immer angegeben werden. Die Messung der Trichloressigsäure-fällbaren Radioaktivität im Markierungsansatz (Trichloressigsäure-Faktor) stellt unseres Erachtens - bei immer gleichbleibender Hormon- und Radiojodmenge - einen 
guten und einfach bestimmbaren Gradmesser für die jeweilige spezifische Aktivität dar. Er gestattet keine Aussage über eine eventuelle Alteration des markierten Hormons durch Oxydations- bzw. Reduktionsmittel oder Autoradiolyse. Erst die prozentuale Bindung an den spezifiischen Antikörper kann darüber Aufschluß geben - und auch das nur hinsichtlich der immunologischen Intaktheit des Moleküls. Wir beobachteten gelegentlich, trotz weitgehender Standardisierung des Markierungsprozesses, erhebliche Schwankungen des Trichloressigsäure-Faktors. Eine Erklärungsmöglichkeit dafür wäre die bekannte Schwankung der ,isotopic abundance" von ${ }^{131} \mathrm{~J}$ zwischen 15 und 30\%. Nach anfänglichen Versuchen mit ${ }^{125} \mathrm{~J}$ zur Markierung von B-TSH und H-TSH beschränkten wir uns zuletzt trotz mancherlei theoretischer Vorteile von ${ }^{125} \mathrm{~J}$ (fast 100proz. isotopic abundance, reine, niederenergetische $\gamma$-Strahlung, längere physikalische Halbwertzeit, höhere Zählausbeute (19)) bei der H-TSH-Markierung auf den Einsatz von ${ }^{131} \mathrm{~J}$ wegen der damit erreichten höheren spezifischen Aktivität und der Beobachtung, daß es zumindest in den ersten 3 Tagen nach der Markierung zu keiner wesentlichen Beeinträchtigung der Versuchsergebnisse durch vermehrten Anfall radioaktiver Degradationsprodukte kommt. Auch mußten wir, ebenso wie andere Autoren (25), die Feststellung machen, daß aus nicht überschaubaren Gründen (chemische Verunreinigungen der ${ }^{125} \mathrm{~J}$-Charge?) gelegentlich bei ${ }^{125} \mathrm{~J}$-Markierungen keine ausreichende Eiweißbindung des Radionuklids erreicht werden konnte (TrichloressigsäureFaktor unter $30 \%$ ). Eine zusätzliche Chromatographie von ${ }^{131} \mathrm{~J}-\mathrm{H}-\mathrm{TSH}$ über Sephadex-G-100 direkt im Anschluß an die Auftrennung über Sephadex-G-75 bringt keine Erhöhung der prozentualen Bindung des markierten Hormons an den Antikörper. Auch die mit ${ }^{131} \mathrm{~J}-\mathrm{H}-\mathrm{TSH}-$ Präparationen vor und nach weiterer Reinigung hergestellten Standardkurven unterschieden sich statistisch nicht voneinander.

Während in unserem B-TSH-System bovines TSH bis zu einer Empfindlichkeit von $5 \mu \mathrm{IU}$ nachgewiesen werden. konnte, zeigten Human Thyrotropin Research Standard A und Plasmen primär hypothyreoter Patienten keine Affinität zum verwendeten B-TSH-Antikörper. Wir konnten damit die Beobachtungen von UTIGER und Mitarbeitern (4) bestätigen, wobei allerdings zu berücksichtigen ist, daß wir nur fünf Antiseren eigener Herstellung zur Verfügung hatten. Sноме und Mitarbeiter $(26,27)$ konnten durch differenzierte immunologische Untersuchungen ein sehr ähnliches Verhalten von $\mathrm{H}$-TSH und denaturiertem B-TSH gegenüber BTSH-Antiseren feststellen. Auch fanden sie, daß die verwendeten H-TSH-Antiseren komplett die biologische Aktivität von B-TSH neutralisierten.

Die unterschiedlichen Ergebnisse der verschiedenen Arbeitsgruppen hinsichtlich der Kreuzimmunität von B-TSH und H-TSH sind am ehesten auf die Verwendung qualitativ verschiedener Antiseren, bedingt durch Immunisierungs-Modus, genetische Vielfalt des immunisierten Tiermaterials usw., zurückzuführen. Ähnliche
Beobachtungen wurden auch bei der radioimmunologischen Bestimmung von menschlichem Parathormon mit Antikörpern gegen bovines Nebenschilddrüsenhormon (28) und bei der Erzeugung spezifischer Antikörper gegen FSH und LH (29) gemacht. Auf jeden Fall sind schon aus Vergleichsgründen spezifische Antikörper der Ausnutzung einer Kreuzimmunität bei radioimmunologischen Bestimmungen vorzuziehen. TSH besteht aus einer Polypeptid-Kette, während zwei solcher Ketten (C I, C II) das LH-Molekül bilden. Die Aminosäurezusammensetzung der CI-Kette von $\mathrm{LH}$ ähnelt sehr der von TSH. Immunologische Studien zeigten, daß Antiseren gegen CI mit LH und TSH kreuzreagieren, während nur eine geringe Kreuzimmunität zwischen dem intakten LH-Molekül und TSH existiert (30). Entsprechend fanden wir im H-TSH-System nur eine geringe, offenbar vom Grad der Verunreinigung durch TSH abhängige Reaktion der H-LH-Präparationen (NIH, Calbiochem) mit dem spezifischen H-TSHAntikörper (NIH). HCG und 2-IRP-HMG zeigten nur eine ganz geringe Kreuzimmunität in höheren Dosisbereichen. Die Zugabe von HCG (10 IU/Probe) zum Reaktionsansatz zur Abbindung gonadotropinaffiner Valenzen des Antikörpers ist folglich beim verwendeten H-TSH-Antiserum des NIH wegen der nur geringen Kreuzimmunität zu den geprüften Gonadotropinen (FSH verhält sich ähnlich wie LH (31)) nicht unbedingt erforderlich. Denn unter physiologischen und pathologischen Bedingungen (Gonadotropin-Spitzen in Cyclusmitte, Menopause; hypergonadotroper Hypogonadismus) werden selten LH und FSH-Spiegel über $100 \mathrm{mIU}$ 2-IRP-HMG gemessen (31). So ergaben sich nach unseren Untersuchungen bei euthyreoten Patientinnen in der Menopause im H-TSH-System ohne HCG-Zusatz auch keine über dem Normbereich liegende TSHSpiegel. Ferner unterscheiden sich Standardkurven (Human Thyrotropin Research Standard A), welche von uns im H-TSH-System mit (10 IU, $100 \mathrm{IU}$ ) und ohne HCG-Zusatz gewonnen wurden, hinsichtlich Verlauf und Empfindlichkeit nur unwesentlich voneinander. Andere Arbeitsgruppen $(32,33)$ berichteten allerdings über gegenteilige Erfahrungen. Unterschiede in der Qualität des verwendeten HCG-Präparates könnten hierbei eine Rolle spielen. Dagegen reagierten im homologen und heterologen H-LH-System (H-LH und HCGAntikörper des NIH bzw. H-LH-Antikörper der Firma Calbiochem) TSH-Präparationen mit hohem und niedrigem H-LH-Gehalt gleichermaßen stark mit den beiden Antiseren, was für eine Kreuzimmunität von $\mathrm{H}-\mathrm{LH}$ und $\mathrm{H}-\mathrm{TSH}$ gegenüber diesen Antikörpern spricht. Es ist also ohne Neutralisation der TSH-bindenden Antikörper-Valenzen nicht möglich, mit den genannten H-LHSystemen luteotropes Hormon bei primär hypothyreoten Patienten zu bestimmen, ohne TSH mit zu erfassen. Im Gegensatz zu LemarCHAND-BÉRAud (32) konnten wir keine Reaktion von Wachstumshormon (bis zu $10 \mathrm{ng} /$ Ansatz) im H-TSH-System (NIH) feststellen. Allerdings verwendeten wir eine andere Wachstumshormon-Präparation. 
Die uns zugänglichen drei H-TSH-Präparationen (Human Thyrotropin Research Standard A, H-TSH des NIH und der Firma Calbiochem) sind zur Erstellung von Standardkurven gut geeignet, ebenso wie der Hypophysenextrakt LER 907 (NIH). Bei unserer Versuchanordnung ist ein genauer gewichts- oder einheitsmäßiger Vergleich der einzelnen Präparationen untereinander nicht möglich.

Wir stellten Untersuchungen zu der Frage an, ob es notwendig ist, den Ansätzen der Standardkurve antigenfreies Plasma (Hypophysektomierten-Plasma) in entsprechender Menge hinzuzufügen, um die Bedingungen der Patienten-Plasma-Proben zu simulieren. Von einigen Autoren (34) wird eine solche Maßnahme gefordert, um zumal im Doppelantikörpersystem nicht näher definierte Fehlermöglichkeiten zu vermeiden. Wir fanden eine etwa 20proz. Abnahme der Antikörperbindung von ${ }^{131} \mathrm{~J}-\mathrm{H}-\mathrm{TSH}$, was zu niedrigeren Ablesungen der Plasmawerte führt, ohne daß der Prozentsatz der unspezifischen Bindung (Kontrolle B) zunahm oder sich der Präzisionsindex der Standardkurven verbesserte. Subtrahiert man im wichtigen niedrigen Meßbereich (bis etwa $20 \mu \mathrm{U} / \mathrm{m} l$ ) von den an einer „Puffer"-Standardkurve abgelesenen Plasmaspiegeln den virtuellen Meßwert eines mitbestimmten HypophysektomiertenPlasmas, so kommt man etwa zu den gleichen Ergebnissen. Bei höheren TSH-Spiegeln fällt diese Differenz kaum ins Gewicht. Wir verzichteten folglich auf einen routinemäßigen Zusatz von TSH-freiem Plasma zu den Ansätzen der Standardkurven. Der Präzisionsindex $\lambda\left(\frac{s}{b}\right)$ als Kriterium für die Qualität von Standardkurven läßt sich für die von uns verwendeten TSH-Systeme nur bedingt anwenden, da die Standardkurven auch im halblogarithmischen System streng genommen einen s-förmigen Verlauf aufweisen. Aus diesem Grunde liegt $\lambda$ bei den von uns errechneten Regressionsgeraden trotz hohem Korrelationskoeffizienten $(r>0,95)$ oberhalb des für radioimmunologische Methoden festgelegten Grenzwertes von 0,1 .

Eines der schwierigsten Probleme bei der radioimmunologischen H-TSH-Bestimmung ist die Festlegung des Normalbereiches, da die untere Empfindlichkeitsgrenze der Methode bei Einsatz der zur Verfügung stehenden
Antiseren (NIH; Calbiochem), im Bereich des TSHGehaltes schilddrüsengesunder Kontrollpersonen liegt. Hinzu kommt, daß in Abhängigkeit von der spezifischen Aktivität des markierten Hormones die TSHWerte zum Teil erheblich schwanken (Markierungen mit einem Trichloressigsäure-Faktor unter $60 \%$ sollten nicht verwendet werden!) und der Variationskoeffizient auch unter Verwendung von Markierungen ähnlicher spezifischer Aktivität bei wiederholter Bestimmung der gleichen Probe in verschiedenen Versuchsansätzen gerade im niederen Konzentrationsbereich $20 \%$ beträgt. Bei $60 \%$ der von uns untersuchten Kontrollpersonen lagen die TSH-Spiegel, unabhängig vom Geschlecht, nach Abzug des Hypophysektomierten-Wertes unter der methodischen Erfaßbarkeit. Andere Arbeitsgruppen $(35,36)$ konnten bei $80-90 \%$ ihres euthyreoten Kollektivs ohne Anreicherung TSH nachweisen. Unser Normalbereich umfaßt unter diesen strengen Maßstäben 2-6 $\mu \mathrm{U} / \mathrm{m} /$; ODELL und Mitarbeiter (14) geben 2,8-9 $\mu \mathrm{U} / \mathrm{m} l$ an. Über den Plasma-TSH-Gehalt bei Patienten mit unbehandelter Schilddrüsenüberfunktion ist wahrscheinlich mit den von uns verwendeten Präparationen und der beschriebenen Methodik keine sichere Aussage möglich. Hiermit in Ubereinstimmung stehen die Ergebnisse anderer Untersucher $(5,8,14,37)$ daß sowohl radioimmunologisch als auch im biologischen Test nach Anreicherung die TSH-Werte im Serum unbehandelter Hyperthyreosen wesentlich niedriger sind als bei euthyreoten Patienten.

Somit erscheint selbst die radioimmunchemische Bestimmungsmethode von H-TSH im homologen System für normale und erniedrigte TSH-Spiegel, in denen eine besondere klinische Relevanz der H-TSH-Bestimmung im Plasma liegt, noch nicht ausreichend empfindlich.

Wir sind Herrn Dr. W. R. Bares, National Pituitary Agency and the Endocrinology Study Section of the National Institute of Arthritis and Metabolic Diseases, Bethesda, Mass., USA., und Herrn Dr. R. Bangham, National Institute for Medical Research, London, für die freundliche Überlassung von spezifischen Antiseren, hochgereinigten Hormopräparationen und Standards sehr verbunden.

Fräulein U. Warnecke, Frau R. LANGer und Fräulein M. HussMANN danken wir für ihre wertvolle technische Assistenz.

\section{Literatur}

1. Werner, S. C. (ed.), Thyrotropin, Ch. C. Thomas, Springfield (Ill.), 1963). USA. - 2. BAKKE, J. L., J. Clin. Endocr. Springfield 25, 455 (1965). - 3. Kтrкhaм, K. E., Endocrinology 25, 259 (1962). - 4. Utiger, R. D., W. D. Odell und P. G. CondLifFe, Endocrinology 73, 359 (1963). - OdeLL, W. D., J. F. WiLber und W. E. Paul, J. Clin. Endocr., Springfield 1179 (1965). 6. Lemarchand-Béraud, Th. und A. Vannottr, Experientia (Basel) 21, 353 (1965). - 7. FreYchet, P., G. Rosselrn und J. Dolars, Proc. Int. Symp. Protein and Polypeptide Hormones ICS 161, p. II, p. 348-350, Excerpta Med. Found., Amsterdam (1968). - 8. OdeLL, W. D., J. F. WILBER und R. D. Utiger, Recent Progr. Hormone Res., N. Y. 23, 47 (1967). - 9. CondLIFfe, P. G. und R. W. Bates, J. biol. Chemistry 223, 843 (1956). 10. McKenzie, J. M., Endocrinology 63, 372 (1958). - 11. v. zUR MühLEN, A. und D. EMrICH, Internat. Atomic Energy Agency,
Wien, 1970. - 12. Greenwood, F. C., W. M. Hunter und J. S. Glover, Biochem. J. 89, 114 (1963). - 13. Berson, S. A., R. S. Yalow, A. Bauman, M. A. Rothschird und K. Newerly, J. Clin. Invest. 35, 35 (1956). - 14. Odeld, W. D., L. VANSLAGER und R. BATES, Radioimmunoassay of human thyrotropin. In: Radioisotopes in Medicine; in vitro studies. U. S. Atomic Energy Commissiom (1968). - 15. National Pituitary Agency - Endocrinology Study Section - and National Institute of Arthritis and Metabolic Diseases, Arbeitsanleitungen: Procedures for radioimmunoassay of HTSH; Procedures for radioimmunoassya of HLH (den Präparationen beigefügt) - 16. Morgan, C. R., R. L. Sorensen und A. LAzarow, Diabetes, N. Y. 13, 579 (1964) - 17. Hales, C. N. und P. J. RANDLE, Biochem. J. 88, 137 (1963). - 18. LorAINE, J. A. und E. T. BELr, Hormone assay and their clinical application. E. \& S. Livingstone, Edinburgh and London, (1966). - 19. Hun. 


\title{
BIOCHIMIE
}

\section{Edité par la Société de Chimie Biologique}

\author{
tel est le titre \\ sous lequel paraîtra à partir de 1971 \\ le „BULLETIN DE LA SOCIÉTÉ DE CHIMIE BIOLOGIQUE“
}

\section{SECRÉTARIAT}

de la Société de Chimie Biologique

J. P. EBEL, Secrétaire Général (Relations Extérieures)

R. PERLES, Secrétaire Général

\section{REDACTION}

F. GROS, Secrétaire scientifique

F. PERCHERON, Secrétaire à la Publication J. NUNEZ, Secrétaire à l'Information

Y. RAOUL, Secrétaire à l'Edition

SECRETARIAT et REDACTION: 4 Avenue de l'Observatoire, PARIS $6^{\circ}$

\section{FASCICULES}

ABONNEMENTS: FRANCE et ZONE FRANC: 150 ffres - BELGIQUE: $1.687,-$ fres - AUTRES PAYS: $186,-$ ffrcs

$$
\text { MASSON et Cie, Editeurs - } 120 \text { Boulevard St Germain - PARIS 6ème }
$$

\section{Titrationen}

in niohtwajsspigen Medien

\section{Von Dr. Istvan Gyenes C. SC. (Chim.)}

Dozent an der Universität für Chemische Industrie Veszprém, Ungarn; Leiter der physikalischchemischen Forschungsäbteilung der Chemischen Fabrik Gedeon Richter AG, Budapest
3., neubearbeitete und ergänzte Auflage Aus dem Englischen übersetzt von Tibor Wein 1970. Mit 206 Abbildungen und 108 Tabellen. 701 Seiten. Format 16,7 ×23,5 cm. Ganzleinen DM 84. ISBN 3432016190

Aus den Besprechungen

... Das Buch ist für alle jene analytisch arbeitenden Chemiker und Pharmazeuten bestimmt, die in der organisch-chemischen und pharmazeutischen Forschung und Industrie tätig sind. Ihnen wird es eine Fülle von Informationen und Anregungen für die Lösung ihrer Analysenprobleme geben. Es sollte schon deshalb in keinem Laboratorium fehlen.

Scientia Pharmazeutica

... Das Werk kann als Standardwerk gelten; es ist modern, akiuell und kann sehr empfohlen werden.

Praxis der Naturwissenschaften 


\title{
ACTA BIOCHIMICA ET BIOPHYSICA \\ ACADEMIAE SCIENTIARUM HUNGARICAE
}

\author{
IIftII \\ Zeitschrift der Ungarischen Akademie der Wissenschaften
}

VOLUME 5

NUMBER 4

Contents

S. Csuzr: Effect of the the Cereus Lytic Enzyme on the Chemical Bonds of the Bacterial Cell Wall

EMILIA CSEKe, L. Boross: Factors Affecting the Reactivity of the Activated SH-Group of D-Glyceraldehyde 3-Phosphate Dehydrogenase

ILMA SzÁsz: Śtructure and Function of Erythrocytes. IV. The Role of Nucleotides and Bivalent Cations in Determining the Shape of Normal and Trypsin Treated Erythrocytes

Ilma SzÁsz, P. Teitel, G. GÁrdos: Structure and Function of Erythrocytes V. Differences in the $\mathrm{Ca}^{2}+-$ Dependence of the ATP Requiring Functions of Erythrocytes

A. Zsindely, M. Szaboles, B. Tankó: Base Composition of Cytoplasmic Ribonucleic Acids Isolated from the Organs of Normal and Brown-Pearce Tumour Bearing Rabbits

A. Zsindely, J. Hutar, B. Tankó: The Use of Diethyl Pyrocarbonate as a Nuclease Inhibitor in the Extraction of Ribonucleic Acids from Animal Tissues

T. DÉvÉNYI: Thin Layer Ion-Exchange Chromatography on Resin-Coated Chromatoplates I. Separation of Aromatic and Basic Amino Acids

T. DÉvénYI: Thin Layer Ion Exchange Chromatography on Resin-Coated Chromatoplates II. Racemization Teśt in Peptide Synthesis

F. FÁbián: The N-terminal Group of Pig Pancreas Amylase (Short Communication)

P. Elödr, Mrlka Krysteva: Structural Investigations on Pancreatic $\alpha$-Amylase. The Effect of Urea on the Ultraviolet Absorption

ERNST, E.: Discrimination of Isotopes by Living Systems. I. Frog Hearts in ${ }^{39} \mathrm{~K}$ and ${ }^{41} \mathrm{~K}$

ERNST, E.: Discrimination of Isotopes by Living Systems. II. Barley Seedlings in $\mathrm{D}_{2} \mathrm{O}$ and $\mathrm{H}_{2} \mathrm{O}$

NiedeTzKy, A., Dalnokr, F.: Discrimination of Isotopes by Living Systems. III. The Effect of Heavy Water on the Motility and Lifetime of Sperms

Trombitás, K.: Submicroscopic Transverse Organization of the Cross-striated Fibril (Preliminary Report)

ACTA BIOCHIMICA ET BIOPHYSICA erscheint vierteljährlich in Heften zu einem Band von etwa 400-500 Seiten. Format: $17 \times 25 \mathrm{~cm}$.

Abonnementspreis pro Band: $\$ 16.00$; DM 64,-

Vertrieb: KULTURA, Budapest 62, Postfach 149; Auslieferung für das Gebiet der Deutschen Bundesrepublik: KUNST UND WISSEN, Erich Bieber, Stuttgart S., Wilhelmstraße 4

AKADÉMIAI KIADÓ, Verlag der Ungarischen Akademie der Wissenschaften, Budapest 502, Postfach 24 
TER, W. M., In: Handbook of experimental immunology., p. 608 to 654. Ed. by D. M. WeIr, Oxford and Edinburgh, (1967). 20. Mrtani, F., R. Conrads, K. M. Bartelt und E. F. Pegriffer, Verh. Dtsch. Ges. inn. Med.343 (1965). - 21. Pennisi, F., J. Nucl. Biol. Med. 12, 137 (1968). - 22. CATt, K., H. D. Niall und G.W. Tregear, A solid phase disc radioimmunoassyay for human growth hormone. J. Laborat. Clin. Med., St.Louis 70,820 (1967). 23. Albert, A., E. Rosemberg, G. T. Ross, C. A. Paulsen und R. J. Rran, J. Clin. Endocr., Springfield 28, 1214 (1968). 24. Bates, R. W., M. M. Garruson, J. A. Cooper und P. G. CondlifFe, Endocrinology 83, 721 (1968). - 25. SelenKow, H.A., Radiol. Clin. N. Am. 5, 317 (1967). -26. ShOME, B., A. F. PARLow, V. D. Ramirez, H. Elrick und J. G. Pierce, Arch. Biochem. Biophysics 103, 444 (1968). - 27. Shome, B., D. M. Brown, S. M. Howard und J. G. Pierce, Arch. Biochem. Biophysics 126, 456 (1968). - 28. Potrs, J. T., L. J. Deftos, R. M. Buckle, L. M.
Sherwood und G. D. Aurbach, In: Radioisotopes in Medicine; in vitro studies. U. S. Atomic Energy Commission, (1968) 207. 29. Odeli, W. D., G. Abraham, H. R. Raud, R. S. Swerdloff und D. A. Fisher, Acta endocr., K'hvn, Suppl. 142, 54. - 30. LiAO, T. H., G. Hennen, S. M. Howard, B. Sgome und J. G. Pierce, J. biol. Chemistry 244, 6458 (1969). - 31. SAXENA, B. B., G. LEYENdecker, W. Chen, H. M. Gandy und R. E. Peterson, Acta endocr. K'hvn, Suppl. 142, 185. - 32. Lemarchand-BÉraud, Th., Acta endocr., K'hvn 64, 610 (1970). - 33. Scriatz, R., Pers. Mitteilung. - 34. ThORELr, J. I., Clin. chim. Acta (Amsterdam) 122, 579 (1968). - 35. Wilber, J. F. und R. D. Utiger, J. Clin. Invest. 48, 2096 (1969). - 36. Hershman, J. M., D. G. ReAd, A. L. Batley, V. D. Norman und T. B. Gibson, J.Clin. Endoct., Springfield 30, 430 (1970). - 37. Adams, D. D., T. H. KenNEDY und H. D. Purves, Proc. Univ. Otago Med. School 46, 55 (1968).

Priv.-Doz. Dr. A. von zur Mühlen Med. Klin. u. Poliklinik 34 Göttingen Humboldtallee 1 